\title{
43945 - ULTRASOUND GUIDANCE WITH CONTRAST ENHANCEMENT LEADS TO SUCCESSFUL BRACHIAL PLEXUS BLOCK
}

\section{Shalini Dhir, University of Western Ontario, London, ON, Canada; Sugantha Ganapathy, University of Western Ontario;}

INTRODUCTION: Ultrasound imaging facilitates brachial plexus localization1 and is known to improve the quality of blocks and onset times 2 .

MATERIAL AND METHODS: Local IRB approval was obtained for this study. A total of 30 patients scheduled for upper limb surgery under regional block were prospectively recruited in this study and evaluated in terms of efficacy, ease of insertion, onset times and primary and secondary catheter failure. Infraclavicular brachial plexus localization, needle and subsequent catheter placement was done posterior to the axillary artery under ultrasound guidance with peripheral nerve stimulation assistance. Needle and catheter tip was identified using hand agitated 5\% dextrose with Doppler color flow on the ultrasound. Onset of block was defined as complete loss of motor and sensory function in the brachial plexus distribution and was evaluated over a 30-minute period. Postoperatively, after the block had worn off, catheter stimulation was attempted and block was re-established under ultrasound imaging.

RESULTS: Mean time to plexus location was $6.5 \mathrm{~min}$, catheter insertion was 4 minutes and onset of complete motor and sensory block was 19.7 minutes. There were no incomplete blocks. Axillary nerve was blocked in $100 \%$ of patients. None of the patients required supplementation or / and GA due to incomplete block. Post operatively, in $87 \%$ of patients brachial plexus could be stimulated. All patients had sensory block and complete analgesia. In $95.7 \%$ of patients spread of drug via the catheter tip could be visualized by hand agitated local anesthetic agent as seen by Doppler on ultrasound.

DISCUSSION: Secondary catheter failure is the main reason why continuous post operative analgesia is incomplete. Contrast enhancement in evaluation of peripheral neural tissue has not been evaluated though it was originally reported in 1968 as a clinical method3. We used ultrasound guidance for location of brachial plexus and insertion of the needle in the infraclavicular area. This has been earlier demonstrated7. We inserted the catheter and demonstrated the position of catheter tip by hand agitated 5\% dextrose by Doppler flow in all patients and achieved 100\% success rate for the initial block. On local anesthetic re-injection, we were able to see spread of the drug under Doppler ultrasound amounting to analgesia in $100 \%$ of patients post operatively. Till date, there is no report of use of ultrasound for positioning of catheter in the infraclavicular area and its confirmation by agitated dextrose / drug with Doppler color flow.

1. Anesth Analg.1997 Oct;85(4)854-7

2. Invest Radiol 1968; 3:356-366.

3. Br J Anaesth 1978; 50:965-7.

4. Reg Anesth Pain Med 2004;29:189-93.

5. Anesth Analg 2005;100:1837-9.

6. Can J Anesth 2005; 52:69-73. 
7. Reg Anesth Pain Med 2003; 28:263-72. 